\title{
1 \\ Broadband Optical Access Technologies and FTTH Deployment in NTT
}

\author{
Hiromichi Shinohara and Tetsuya Manabe \\ NTT Access Network Service Systems Labs, Ibaraki, Japan
}

\subsection{INTRODUCTION}

The NTT access network encompasses NTT central offices and customer terminals and networks, and consists of transmission devices for individual customers in each NTT central office, transmission lines, and corresponding devices on the customers' premises. Unlike trunk relay lines between NTT central offices, access networks are not designed to carry concentrated communication traffic. Since cost reduction strategies such as multiplexing and task splitting cannot be employed, it is important that the component parts are as inexpensive as possible.

Providing an access network for ordinary customers that covers the length and breadth of the nation represents an enormous undertaking. For instance, the process of upgrading facilities to alleviate congestion in telephone services necessitated a substantial 20-year investment program that started in the 1950s, when NTT was known as the Nippon Telegraph and Telephone Public Corporation. Given the scale of the task, it is especially important to develop simple, well-designed technologies and systems for the construction, operation, and maintenance of the access network.

The transmission characteristics of the access network have a direct bearing on service provision. Equipment and system designs must strike an appropriate balance between the need to keep costs down at the initial stage and the need for the scalability and flexibility to accommodate the future diversification and modernization of the services.

Thus, the access network is governed by a wide range of conditions and a different level of requirements. Much is expected from NTT's optical technology research program, which began with the trunk network. In particular, it is hoped that the optical

Broadband Optical Access Networks and Fiber-to-the-Home: Systems Technologies and Deployment Strategies Chinlon Lin (C) 2006 John Wiley \& Sons, Ltd 
technology will provide NTT with greater flexibility to respond to the very rapid changes in the marketplace that followed privatization in 1985. These changes have included the introduction of competition, which has forced prices down; the shift towards mobile phones at the expense of landline services, which has affected equipment investment; and the paradigm shift brought about by the advent of Internet and broadband services.

The technologies involved in access networks are many and varied. They include optical transmission systems and optical cable and associated hardware, optical wiring based on demand and environmental considerations, and construction, cable laying, and maintenance systems. These technologies have evolved in line with changes in demand patterns and the socio-economic environment. Similarly, NTT's equipment investment and service development strategies are both influenced by technological progress.

This chapter provides a brief overview of the development of optical access technology in Japan, before moving on to a discussion of modern broadband services. Finally, it will examine the current state of optical access systems used in broadband services, and consider the use of access network technology in the broadband services of the future.

\subsection{HISTORY OF OPTICAL TECHNOLOGY IN JAPAN \\ 1.2.1 THE FIRST RESEARCH ON SUBSCRIBER OPTICAL TRANSMISSION SYSTEMS}

Research into optical transmission technology for subscriber systems and existing access systems began in 1977, and research into trunk relay optical transmission technology began shortly thereafter. Trunk relay optical transmission research was geared towards such objectives as multiplexing, long-distance transmission, and relay-free system design. On the other hand, the goals of optical transmission technology for access systems were to achieve overall economic targets, design outdoor facilities whose simplicity of construction and operation made them suitable for mass production, and develop services that fully utilize the benefits of optical access networks.

\subsubsection{FROM MULTI-MODE FIBER TO SINGLE-MODE FIBER}

Multi-mode fiber was originally used as subscriber optical fiber. In 1988, NTT decided to introduce single-mode fiber, which had originally been developed for trunk networks, into the optical access network as well. This decision followed research into the best way to introduce the fiber into the subscriber network [1].

The decision to go with the potentially more costly single-mode fiber in the subscriber system, where cost considerations were paramount, was indeed a bold one, especially considering that most other countries were considering multi-mode fiber for subscriber systems. However, given the low-loss characteristics of single-mode fiber and its suitability for broadband applications, it was definitely the correct decision for the future. The introduction of single-mode fiber was made possible through the development of new technology in a number of areas. In particular, these developments related to high-precision 
manufacturing (using a process known as total synthesis), the more economical production of long-wavelength light sources, photoreceptors, and fiber interconnection and installation techniques that enabled optical fiber cable to be laid more efficiently. There were also significant economies of scale to be derived from the standardization of fiber with the trunk network.

The focus of research subsequently shifted to more advanced areas, including simple, lowcost wiring configurations such as multi-core cables, thinner wires and multi-core optical fiber connectors; low-maintenance, remote-operated gas-less systems; and fast, low-cost multi-core alternatives.

\subsubsection{DEVELOPMENT OF CT/RT SYSTEM}

Research into the use of optical technology in ordinary subscriber telephone services began in the late 1980s with the Central Terminal/Remote Terminal (CT/RT) system, which is employed in the section of the network equivalent to a feeder line. CT/RT delivers economies of scale through subscriber multiplexing, and allows systems to be scaled to suit line capacity. It also permits the diversification of services into areas such as Integrated Services Digital Networks (ISDN) and dedicated service support. As the results of research and development began to translate into cost reductions, it became possible to design smaller RT cabinets suitable for installation along roadways, on telegraph poles and inside buildings. The first indoor model, installed at the Chiyoda pilot plant in Tokyo in February 1993, featured 1000-strand multi-core optical fiber cable. The CT/RT system, which was later named FALCON, was deployed nationwide in Japan as a typical optical access system.

\subsubsection{MOVING TOWARDS FTTH}

Fiber-To-The-Home, or FTTH, cannot be achieved until we develop new forms of technology capable of both delivering substantial cost reductions across the board and facilitating a seamless transition from metal to optical cable systems. FTTH will require advances in a range of areas from hardware, such as cables and transmission system components, to nonhardware items, such as network design, construction, operation, and ongoing maintenance. Following the successful development of CT/RT, NTT continued research and development with the goal of realizing FTTH.

The Passive Double Star (PDS), which makes it possible for NTT central offices and multiple customers to share cables, was considered particularly promising with respect to cost reduction. The system consists entirely of passive components, and so unlike the CT/RT system it does not need an electricity supply (or the associated space requirements). Furthermore, the same cost-sharing benefits as the CT/RT system are complemented by impressive cost reductions in terms of the components used at the RT end. Another reason for the appeal of PDS was the network topology, which provides excellent compatibility with distribution services such as CATV.

A number of two-way communication systems were developed, including Synchronous Transfer Mode PDS (STM-PDS) [2,3], Asynchronous Transfer Mode PDS (ATM-PDS) [4-7], and Sub-Carrier Multiplexing PDS (SCM-PDS) [8,9]. 


\subsubsection{OPTICAL SYSTEMS AT METAL-WIRE COSTS}

In 1994, NTT announced its intention to accelerate the development of optical fiber access networks and provide an upgraded communication infrastructure for future broadband expansion. To achieve the objectives set out in the published statements, it would be necessary to achieve further economies with FTTH technology. To this end, in 1995 NTT embarked on research into cost reduction initiatives, particularly in relation to distribution systems and user-end optical distribution line systems. When the research program began, the cost per subscriber of optical access was some six to eight times greater than that for access via conventional metal wire; by 1997-1998, this had been reduced to approximately double the cost, and by 2000 the costs were the same. The core technological developments that enabled NTT to achieve its cost-reduction objectives were based on five key concepts:

(1) new approach to network configurations;

(2) low-cost optical PDS;

(3) low-cost optical fiber cables and associated technology;

(4) simplicity of installation and maintenance; and

(5) optical operation systems.

The newly developed technology first appeared in the optical access system, commonly known as the $\pi$ system [10], which essentially involved grafting an optical access network onto an existing metal-wire telephone infrastructure. The optical network was brought as close as possible to subscriber homes (usually to the nearest telephone pole or the outer wall of an apartment building). It was terminated at a $\pi$-ONU device carrying the traffic of 10-20 subscribers, which was then linked to the existing metal cables. In July 1996, NTT announced its plan to deploy the $\pi$ system to upgrade the access network.

\subsubsection{ACCESS NETWORK OPTICAL UPGRADING PROGRAM}

The construction of an optical access network requires a huge investment. It is therefore necessary to prepare a proper equipment investment program that takes account of service supply and demand considerations. In addition to upgrading metal-wire equipment to optical fiber, efforts were made to introduce technology developed for new services tailored to meet unknown demand levels and business requirements. The combination of the $\pi$ system and optical fiber cable at the same cost as metal cable meant that optical access systems could also be extended to ordinary user districts where the upgrading of aging metal cable equipment was required.

\subsection{TRENDS IN BROADBAND SERVICES}

\subsubsection{GROWTH OF BROADBAND SERVICES IN JAPAN}

We first describe the trend of broadband services in Japan. Figure 1.1 shows the recent growth in the number of broadband users in Japan. We have three categories of broadband communications. The dominant broadband medium is the ADSL service with more than 13.6 million users. CATV comes next, but its growth is slackening off, and has become 


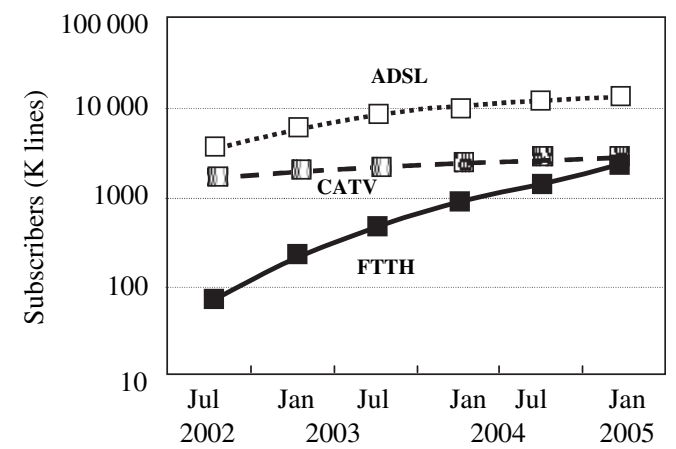

Figure 1.1 Increase in demand for high-speed Internet access. Source: Ministry of Internal Affairs and Communications, JAPAN.

nearly flat. The last is FTTH, which is growing rapidly and the number of users has exceeded 2.8 million and is still growing. Figure 1.2 shows the quarterly increment in new broadband access users. The number of new FTTH users surpassed the number of new ADSL for the first time in the first quarter of 2005. This is the Japanese network environment for which we are now developing technologies.

Another issue with the FTTH service is so-called 'regional disparities.' Although ADSL broadband services are steadily spreading throughout Japan, FTTH (which brings optical fiber directly to the home) is still confined to Tokyo and Osaka and their immediate surroundings, with little penetration into regional cities. Research and development programs will also need to focus on the issue of eliminating regional disparities with respect to broadband services.

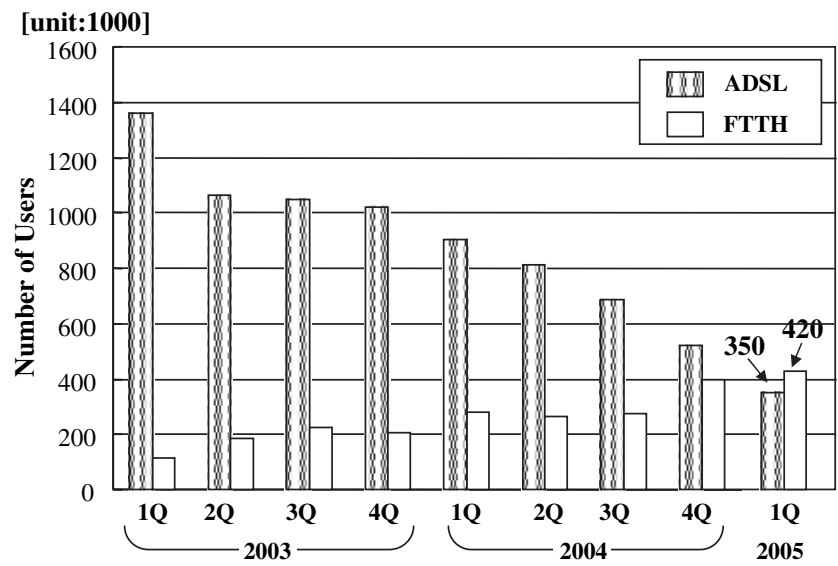

Figure 1.2 Quarterly increment in new users. Source: Ministry of Internal Affairs and Communications, JAPAN. 


\subsubsection{VISION FOR A NEW OPTICAL GENERATION}

\subsubsection{Broadband Leading to the World of Resonant Communication}

In November 2002, NTT released a statement entitled 'Vision for a new optical generation: Broadband leading to the world of resonant communication,' describing its outlook for the ubiquitous broadband era of around 2008 based on optical technology [11].

In the optical fiber 'resonant communication environment' envisaged by NTT for the year 2008, video and related technology will provide more natural, real-time communication than has been possible with narrowband, thereby achieving the ultimate aim of communication, which is to demolish the barriers of time and distance. Breaking down the time barrier translates into more free time for people, while breaking down the distance barrier will allow individuals and corporations to expand their spheres of activity. This in turn promotes human activity on a global scale, transcending national and regional boundaries as well as industries and generations, and enables available knowledge and wisdom to be shared among individuals, companies, and borderless business operations. In this way, individuals and corporations will be able to access knowledge from around the world in what is termed the 'cooperative creation of knowledge.' This will revolutionize both the way we live and the way we do business.

The announced vision has been adopted as a common theme for the entire NTT Group, which is working to develop advanced business models by stimulating demand for optical services and creating business solutions. At the same time, partnerships with both domestic and overseas businesses in a variety of different fields are helping to promote and develop the resonant communication environment in industry.

\subsection{OPTICAL ACCESS TECHNOLOGY BEHIND BROADBAND SERVICES}

In this section, we describe the access network technology that is currently used to provide broadband services. The key requirements of the access network are economy, performance, and ease of use for both installation technicians and customers. Ease of use has a direct bearing on cost, since a well-designed network is easier and hence less costly to set up and maintain. And lower network setup and operating costs mean that services can be provided in both rural and metropolitan areas, thereby reducing regional disparities. Below, we discuss some of the technologies that have been developed with these objectives in mind.

\subsubsection{OPTICAL ACCESS TECHNOLOGY FOR CURRENT BROADBAND SERVICES}

\subsubsection{Optical Access Systems}

Figure 1.3 shows the configuration of an optical access network. Optical technology provides an economical way to transmit large volumes of data. Thus, an optical fiber system extends from NTT substations to points near customers' homes, such as footpaths and telephone poles. At the telephone pole or its equivalent, optical signals are converted into electrical signals, which are then fed down metal cables to individual households. 


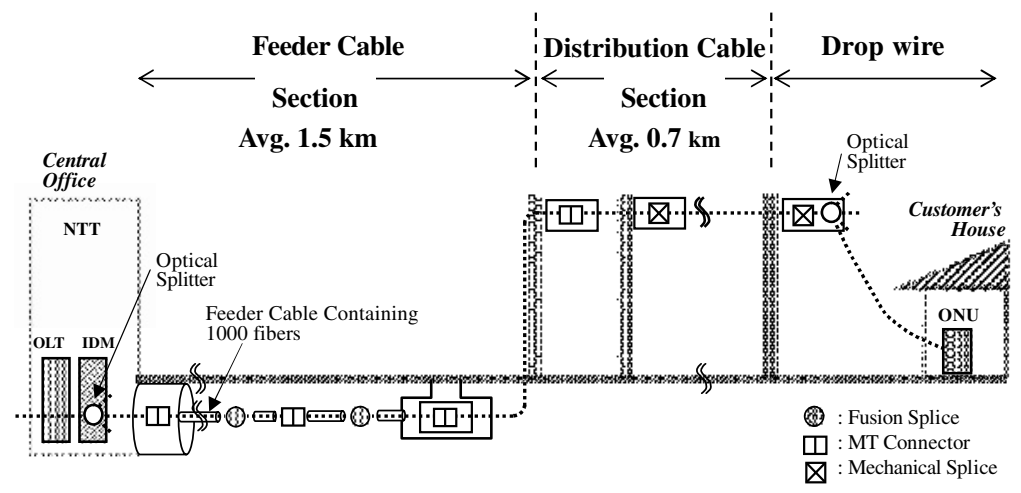

Figure 1.3 Overview of NTT's optical access network.

With FTTH, optical fiber is connected right through to customers' homes to provide faster broadband services. The FTTH system shown in the diagram employs the PON configuration, where a signal from one device at a substation is used by many customers. The signal is distributed to different customers via an optical coupler, which is a simple low-cost device made from glass. There are substantial economic benefits when a single substation device can service multiple customers.

NTT has finished developing a carrier-grade Gigabit EPON system for commercial deployment, as shown in Figure 1.4. The GE-PON system [12,13] is one example of an optical access system based on FTTH in a PON configuration. It provides a transmission capacity of $1 \mathrm{~Gb} / \mathrm{s}$ through optical fiber at the substation and has an Ethernet interface. Key technologies provided by the GE-PON system include encryption and burst reception, both vital components of the PON setup, and bandwidth control and delay suppression, which are used to maximize system performance. NTT is also involved in the development of key carrier-grade operation functions.

Figure 1.5 is a diagram showing encryption technology. Encryption technology is employed during transmission from a substation to ONU devices in customers' homes. In a PON system, an optical star coupler is used to split the optical signal from the substation to the ONU devices. Since the entire signal reaches all ONU devices, a different form of encryption is used for each ONU device. This ensures that each ONU reads only signals intended for that customer.

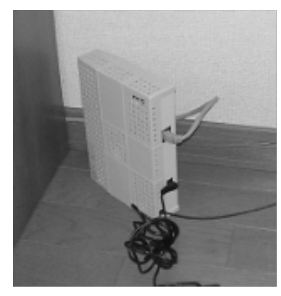

ONU

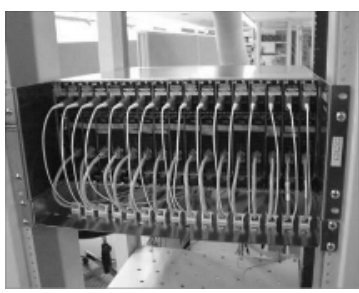

OLT

Figure 1.4 Photographs of commercial gigabit EPON (GE-PON) system. 


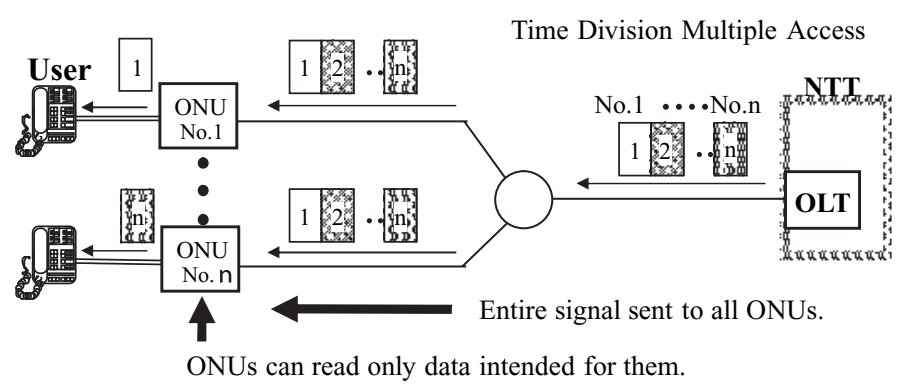

Figure 1.5 Downstream signal encryption.

Figure 1.6 is an illustration of burst transmission technology, which is employed during the transmission of an upstream signal from an ONU to an OLT. One function of burst technology is to prevent collisions between signals sent from customers' homes. A single receiver at the OLT accepts signals from multiple ONU devices. Since different signals cannot be received simultaneously, times for transmitting signals to the OLT are allocated to the ONU devices, and collisions are avoided by processing these transmissions in a set order.

Also, the different lengths of optical fiber between the coupler and the various ONU devices generate differences in path loss. This means that although each ONU device generates optical signals at the same strength, by the time the signals reach the OLT the power levels will vary somewhat. The receiver must be capable of processing signals of various strengths in rapid succession. This is achieved through the use of burst transmission technology.

Now let us look at some of the technologies used to maximize the performance of the PON system. The first is bandwidth control. Figure 1.7 shows the dynamic bandwidth allocation. Different users require different bandwidths at different times. Sometimes they need to transfer large amounts of data, while at other times they may need to transfer only a little. Rather than allocating the same bandwidth to every user, the system makes its 'best

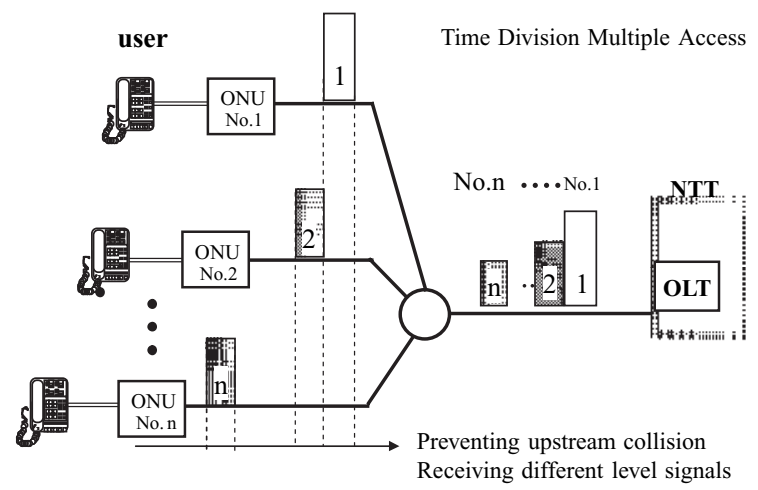

Figure 1.6 Receiving burst signals from different ONUs. 


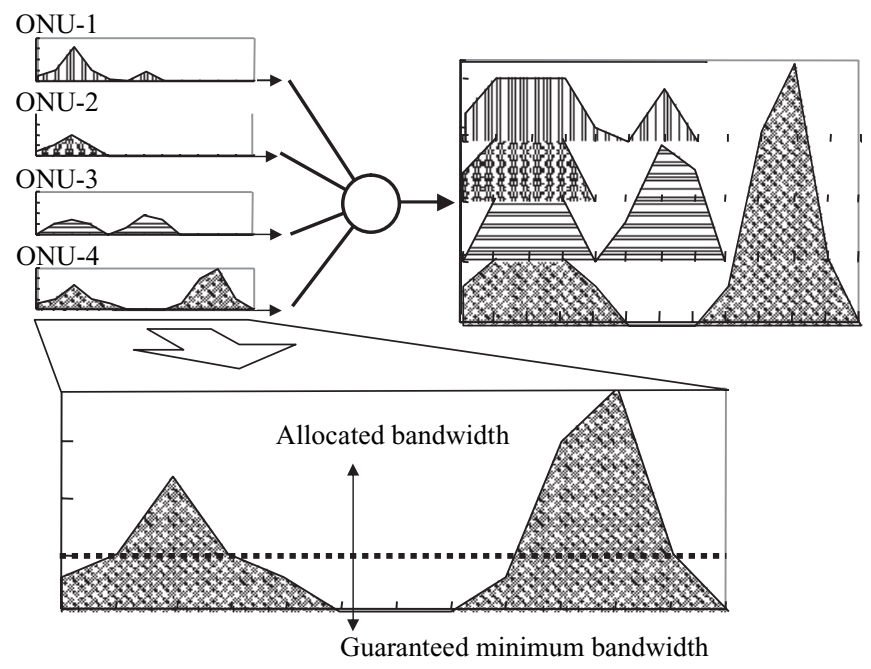

Figure 1.7 Dynamic bandwidth allocation.

effort' to redirect unused bandwidth to users who need it the most. In this way, all users are guaranteed a minimum level of bandwidth, and are also provided with additional bandwidth when required, which is allocated economically on a best-effort basis.

The priority control, shown in Figure 1.8, is another form of technology used to maximize the PON system performance. Video and audio data must be transmitted in real time. GEPON gives priority to the regular transmission of signals associated with such services, and transmits other signals as time permits. Information classified as 'minimum delay class' is given priority, with regular transmissions at shorter cycles, while information classified as 'normal delay class' has lower priority. These functions are controlled equitably on a besteffort basis in order to provide users with an appropriate environment in which to enjoy audio and video services.

The technique of wavelength division multiplexing (WDM) significantly increases the transmission capacity of optical fiber by enabling signals with different wavelengths to be transmitted together along the same fiber. The ITU-T has established standards for the WDM of up- and downstream signals and video signals in each optical fiber in an optical access line. In accordance with these standards, the upstream line from the ONU to the substation

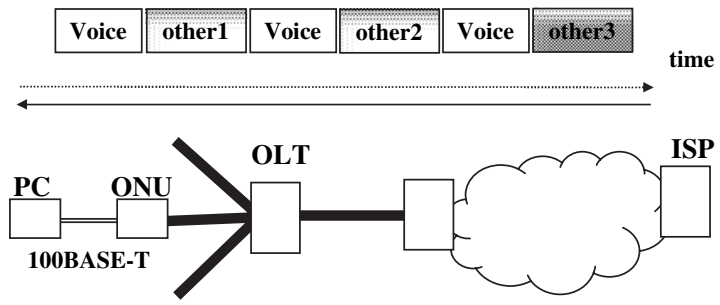

Figure 1.8 Low-delay transmission class applied (for example) to voice signals. 
uses the $1.3 \mu \mathrm{m}$ wavelength band, while the downstream line from the substation to the ONU uses the $1.48-1.5 \mu \mathrm{m}$ wavelength band. For video signals, the standards specify up- and downstream lines that use different wavelengths in the $1.55 \mu \mathrm{m}$ band. In this way, a single optical fiber can be used to carry upstream, downstream, and video signals, using prism-like optical elements (coarse wavelength division multiplexing or CWDM elements) to split and then recombine the various wavelengths.

NTT has developed the STM-PON, B-PON, and GE-PON systems to meet the increasing transmission capacity requirements. In light of the anticipated need for even greater capacity in the future, NTT continues to work on the development of new systems designed to harness the broadband capabilities of optical fiber.

\subsubsection{Optical Access Installation}

In order to provide FTTH services to customers at affordable prices, it is necessary to reduce both the cost of optical access systems and the expenses associated with the installation of optical fiber and associated hardware by simplifying the installation of optical drop cables, optical cabinets, and indoor wiring. NTT is working on developments in a number of areas designed to improve the installation process.

One example is bend-resistant optical fiber. Conventional optical cable requires a minimum bending radius of $30 \mathrm{~mm}$ during installation to avoid damage. NTT has developed an optical cable that can be bent at $15 \mathrm{~mm}$ without fear of damage. Figure 1.9 shows photographs of conventional and newly developed bend-insensitive indoor optical fiber cables. Not only is the cable easier to work with, it has a cleaner look since the bent sections are less prone to swelling. This improvement will have a significant impact on the installation of optical cable in ordinary homes. Furthermore, the introduction of a curled form of optical fiber with a low bending loss makes the cable as easy to handle as metal wire cable such as telephone receiver cords. Curled cable will greatly facilitate the ONU connection procedure shown in Figure 1.10 [14].

The installation of the optical cabinet used for the optical drop cable has been made easier. Figure 1.11 shows photographs of conventional and newly developed cabinets [15]. It is no longer necessary to provide a length of fiber with its sheath removed at the fiber termination. A length of exposed optical fiber must be provided at the end of the drop cable for connection to the indoor optical cord via a mechanical splice. The optical fiber can be connected directly to the drop cable using a special connector, and this can be accomplished

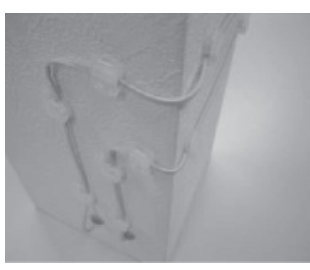

Bending radius: $15 \mathrm{~mm}$

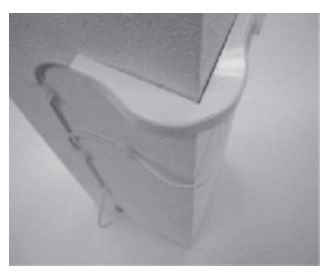

Bending radius: $30 \mathrm{~mm}$

Figure 1.9 Photographs of newly developed bend-insensitive (left) and conventional (right) indoor optical fiber cables. 


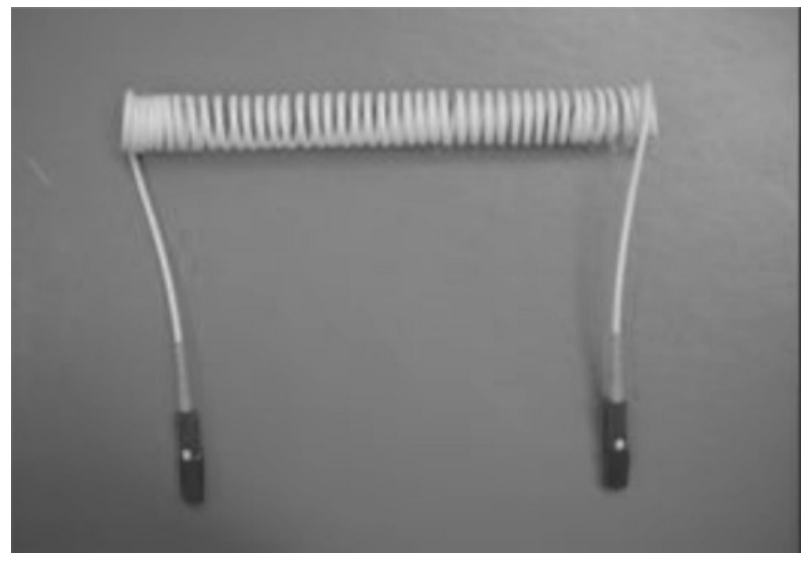

Figure 1.10 Photograph of flexible curled cable.

on site during the wiring process, eliminating the need for exposed optical fiber cord. Meanwhile, the bend-resistant fiber cord has reduced the required length from 30 to $15 \mathrm{~mm}$. As a result, the volume of the new optical cabinet is around one-third that of the conventional cabinet.

Conventional drop cable contains metal wire as a reinforcing tension member, and this needs to be grounded to protect against, for example, lightning. The new cable uses nonconducting Fiber Reinforced Plastic (FRP) as the reinforcing tension member. Grounding is no longer required and this reduces the time needed for the installation of lead-in wires by around $20 \%$.

\subsubsection{Wireless Access}

In some cases it is not easy to service the demand for broadband access. Laying new optical fiber throughout existing apartment buildings, for instance, can present difficulties. WIPAS [16], a wide-area FWA system, was developed as a means of providing broadband services in such situations. Figure 1.12 shows the WIPAS system. Access is provided from a small wireless base station mounted on a telephone pole, which transmits wirelessly to outdoor

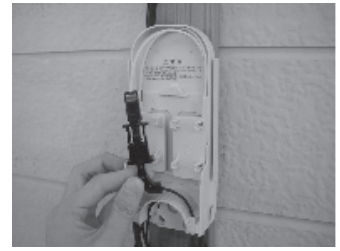

Newly developed cabinet.

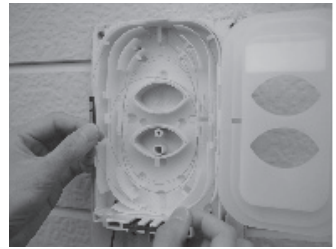

Conventional cabinet.

Figure 1.11 Photographs of newly developed (left) and conventional (right) optical connector cabinets. 


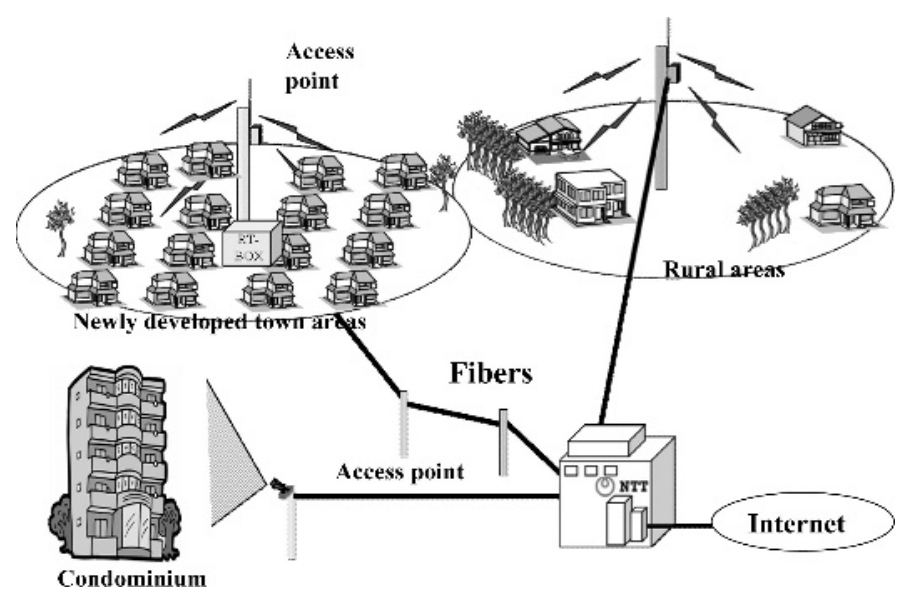

Figure 1.12 Wireless IP access system (WIPAS).

terminals usually installed on the verandas or rooftops of user households. WIPAS provides an inexpensive way to deliver broadband services tailored to local conditions in areas lacking a fully developed optical fiber network. In this way, wireless technology can be used to supplement FTTH in certain circumstances and provide a quick and inexpensive alternative source of broadband services.

\subsubsection{BROADBAND ACCESS NETWORK TECHNOLOGY IN THE FUTURE}

This section discusses the future of access technology. The target of the Medium-Term Management Strategy of the NTT Group is to provide optical access to 30 million customers in Japan by 2010. To achieve this target, it will be necessary to deliver further improvements in cost, performance, and ease of handling. We shall now examine these parameters with respect to optical access systems, optical access installation, and wireless systems.

\subsubsection{Optical Access Systems}

The primary development objective for optical access systems is performance enhancement, particularly with respect to cost, handling, and capacity. In the preceding section, we discussed optical systems that use different wavelengths for the up- and downstream communication lines. It should be possible to boost the cost-effectiveness and performance further by utilizing the broad optical wavelength domain of optical transmission routes via techniques such as WDM, which increases transmission capacity by transmitting multiple wavelengths through a single fiber. In terms of ease of use, systems can be designed to utilize existing networks and facilities. This approach minimizes installation costs and makes it easier to introduce and add new services. In addition, the introduction of common specifications for optical interface modules promotes component sharing for improved economy and user-friendly design. 
There are two ways to harness the broad optical wavelength domain of optical fiber: wavelength division multiplexing, WDM, as described above, and code division multiple access, CDMA, which is already used for mobile telephone systems. We begin by looking at the general features of WDM in access systems.

The only extra initial investment required for WDM, in addition to the optical transceiver and transmission paths used in conventional systems, is the CWDM (coarse WDM) unit. There are two types of CWDM unit: one which splits light from the reception side into multiple wavelengths, and the other which combines optical signals of different wavelengths from the transmission side. Normally, the aim is to keep initial investment to a minimum, since there is rarely any demand for high-volume data transmission capacity at the outset. Ideally, the system design should be able to accommodate future capacity expansion in line with demand growth without great expense. WDM provides this feature. In most cases it is possible to expand capacity simply by adding more optical transceivers; there is no need to lay additional optical fiber. Similarly, if the original design capacity of $100 \mathrm{Mb} / \mathrm{s}$ is insufficient, it can be upgraded to $1 \mathrm{~Gb} / \mathrm{s}$, for instance, simply by installing a new $1 \mathrm{~Gb} / \mathrm{s}$ transceiver. The changeover can be performed without affecting systems operating at other wavelengths.

The same advantages apply equally in the case of access systems, making the future expansion of system capacity an inexpensive proposition. WDM can be used in access networks as an economical means of boosting transmission capacity by adding different wavelength transceivers, but it also promises economic benefits in other areas. For instance, if demand for Internet services is accompanied by demand for video and other content delivery services, these new services can be accommodated on new wavelengths over the existing optical fiber. It is also possible to allocate separate wavelengths to users who require greater transmission capacity. In this way, WDM represents an economical way to utilize existing networks, allowing the flexibility to upgrade performance and functionality by increasing capacity and providing new services.

Thus, WDM makes maximum use of existing networks and equipment, and provides an economical means of delivering advanced services simply by upgrading terminals as required. The underlying system concept presupposes compatibility with existing networks and equipment. Next, we consider economic and ease of handling issues as regards WDM equipment and components.

Conventional WDM devices consist of integrated sets of electrical and optical components for each separate wavelength, gathered together on a board. With this approach, the specifications for each wavelength are slightly different. Fabrication costs are higher, since it is necessary to produce a large number of slightly different devices to make up a single unit.

In contrast, with the new devices the interfaces between the optical transceiver, electrical processor, and CWDM are common to all manufacturers, and are designed as interchangeable modules. This approach allows the mass production of fewer components for less total cost. Furthermore, the optical transceivers are integrated into the body of the unit, making the entire relay both smaller and cheaper to produce. The same principles also apply to the CWDM module [17-19].

NTT is using this approach to develop WDM optical modules designed to reduce the cost of WDM systems. The optical transceiver modules used to emit and receive light at various wavelengths and the CWDM modules are designed to be readily interchangeable and thus 
allow greater flexibility in terms of system configuration. For instance, it is possible to design a point-to-point transmission system, as used for relay transmission, consisting of the light-to-electric conversion of the user signal, an electrical processor, electric-to-light conversion, and an optical fiber transmission path. A wavelength converter can be configured with similar ease. The cascade format, used extensively in LAN design, can be configured to generate a number of different wavelengths (normally at the server), with successive repetitions of the reception, transmission, splitting, and recombining of different wavelengths for each user.

Further into the future, we can envisage the use of CDMA technology in optical communication systems. The advantages of CDMA are already well known in relation to mobile telephone systems. First, the code division format offers excellent resistance to signal interference, or in the case of optical communication, to light interference. Thus, if a customer inadvertently introduces a wavelength into the PON network that acts as interference, this will have only minimal impact on other customers. As with wireless communication, CDMA is a more efficient use of the available wavelengths (spectrum) than WDM. This technology could potentially prove vital in the event of a shortage of optical fiber bandwidth, as it will help us to make better use of the available wavelengths. Also, allocating codes rather than separate wavelengths to individual users allows more flexibility in planning user deployment.

In this way, the next-generation GE-PON, in the form of systems such as WDM that are designed to utilize the broadband capabilities of optical fiber, will deliver economic and performance benefits and be easier to handle.

\subsubsection{Optical Access Installation}

We now consider optical access installation technology. By creating small holes in the fiber, it is possible to change the fiber properties [20,21]. This technology enables to develop fiber with a very small bending radius and substantially reduced bending losses. The configuration and bending loss characteristics of this fiber, which is known as Hole-Assisted Fiber, is shown in Figures 1.13 and 1.1.4, respectively. Because such a fiber could easily be bent with a radius of less than $15 \mathrm{~mm}$, fiber cords containing such fiber could be laid quickly indoors in any required configuration without compromising aesthetic requirements.

\subsubsection{Wireless Access}

Finally, we briefly discuss the wireless access technology used to complement optical access services. Wireless systems on the so-called 'last one mile' segment are capable of

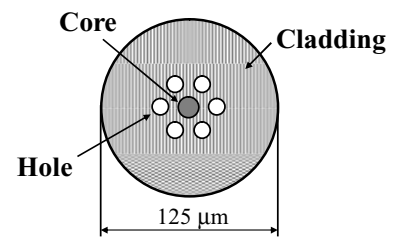

Figure 1.13 Configuration of hole-assisted fiber. 


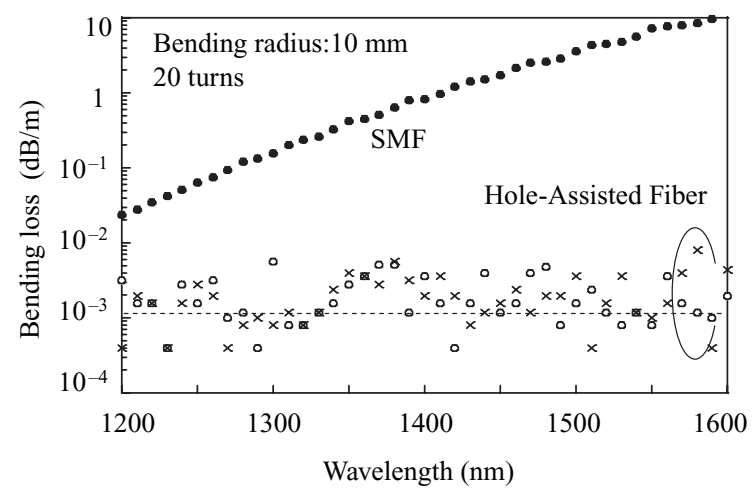

Figure 1.14 Bending loss characteristics of hole-assisted fibers.

transmission speeds of $50 \mathrm{Mb} / \mathrm{s}$, which is about half that of optical drop fiber operating at $100 \mathrm{Mb} / \mathrm{s}$. The aim is therefore to increase this speed to that of optical fiber. The deployment of additional base station antennas must not be too expensive, since this will occasionally be needed to ensure quality of service. In addition, it will of course be necessary to reduce the overall costs. Future development of wireless access technology is geared towards these objectives.

\subsection{CONCLUSION}

In this chapter, we briefly reviewed the history of optical communication systems in NTT, Japan. In terms of broadband services, the current focus of research and development is on servicing a range of user demands and reducing regional disparities in service levels. We discussed optical access technology for broadband services in the form of the GE-PON system, installation technology, and wireless access technology, and analyzed NTT's research and development with respect to the broadband environment. Finally, we looked at the development of network access technologies for future broadband services.

Such factors as the increasing interest in high-volume applications including Internet video distribution services and large data file exchange, the expectations of guaranteed services to complement existing best effort services, and the ongoing diversification of the network environment emphasize the need for the further development and enhancement of transmission services. Access networks represent the entrance through which customers gain access to such services. As such, more effort must be poured into the research and development of optical access networks in line with the advent of ever more advanced broadband services tailored to the market demand.

\section{REFERENCES}

1. Shimada S, Hashimoto K, Okada K. Fiber optic subscriber loop systems for integrated services: the strategy for introducing fibers into the subscriber network. J Lightwave Tech, 1987; LT-05: $1667-1675$. 
2. Fujimoto Y, Ohtaka A, Yamaki K, Miki N. STM Shared Access System for High-speed IP Communications. Proc. European Conf. on NOC 2000, June 2000, pp. 110-117.

3. Yoshino M, et al. DBA Function for Broadband Passive Optical Network Systems. Proc. OHAN/FSAN 2001, April 2001, pp. 3.1-1-3.1-8.

4. Shibata Y, Okada K. Development of FTTH/B B-PON System in NTT. OHAN/FSAN Workshop 2001 F7, April 2001.

5. Ford B. Development of Fiber to the Home. OHAN/FSAN Workshop 2001 F5, April 2001.

6. Laurette M, Abiven J, Durel S. France Telecom FTTH/O Developments and Plans. OHAN/FSAN Workshop 2001 F6, April 2001.

7. Ueda H, Okada K, Ford B, Mahony G, Hornung S, Faulkner D, Abiven J, Durel S, Ballart R, Erickson J. Deployment status and common technical specifications for a B-PON System. IEEE Com Mag, 2001.

8. Way W I. Subcarrier multiplexed lightwave system design considerations for subscriber loop applications. J Lightwave Tech 1989; 7(11): 1806-1818.

9. Kikushima K, Yoneda E. Erbium-doped fiber amplifiers for AM-FDM video distribution systems. IEICE Trans 1991; E74(7): 2042-2048.

10. Ikeda K, et al. Development of New Optical Access System. 8th International Workshop on Optical/Hybrid Access Networks, Atlanta, 1997.

11. Vision for a New Optical Generation - Broadband Leading to the World of Resonant Communication -, NTT News Release, 11/25/2002, (http://www.ntt.co.jp/news/indexe.html)

12. Ochiai K, Tatsuta T, Tanaka T, Yoshihara O, Oota N, Miki N. Development of a GE-PON (Gigabit Ethernet Passive Optical Network) System, NTT Technical Review, Vol. 3, No. 5, May 2005, pp. 51-56.

13. Abrams M, Becker PC, Fujimoto Y, O'Byrne V, Piehler D. FTTP Deployments in the United States and Japan - Equipment Choices and Service Provider Imperatives. IEEE/OSA J Lightwave Tech, Jan. 2005; 23(1): pp. 236-246.

14. Hiramatsu K, Kurashima T, Araki E, Tomita S. Development of Optical Fiber Curl Cord. Proc of Comm Conf IEICE, B-10-3, pp. 374. March 2004.

15. Aoyama H, Tanaka H, Hoshino Y, Oda Y. Optical Wiring Technology for Home Networks for a Service-ready and Low-cost FTTH Service. NTT Technical Review, Vol. 3, No. 4, April 2005, pp. 33-37.

16. Nidaira K, Shirouzu T, Baba M, Inoue K. Wireless IP Access System for Broadband Access Services. Int Conf Comm IEEE, Vol. 6 WC15-1, June 2004, pp. 3434-3438.

17. Small Form Factor Pluggable (SFP) Multi Source Agreement, INF-8074, http://www.schelto.com/SFP/

18. Kimura H, Yoshida T, Kumozaki K. Compact PLC-based Optical Transceiver Module with Automatic Tunable Filter for Multi-rate Applications. IEE Electron Lett 2003; 39(18): 1319-1321.

19. Yoshida T, Kimura S, Kimura H, Kumozaki K, Imai T. New 156M/2.5Gbit/s Multi-rate SFP Transceiver with Automatic Sensitivity Switching. Proc 10th OptoElectron Comm Conf (OECC 2005), July 2005, pp. $204-205$.

20. Tajima K, Nakajima K, Kurokawa K, Yoshizawa N, Ohashi M. Low-loss Photonic Crystal Fibers. Proc Optical Fiber Comm Conf (OFC 2002), ThS3, 2002; pp. 523-524.

21. Nakajima K, Hogari K, Zhou J, Tajima K, Sankawa I. Hole-assisted fiber design for small bending and splice losses. IEEE Photon Tech Lett 2003; 15(12): 1737-1739. 